\title{
Optical pumping of the atom magnetic moment under spin polarization equal to zero
}

\author{
Evgeniy Popov ${ }^{1, *}$, Sergey Voskobojnikov ${ }^{2}$, Konstantin Barantsev ${ }^{1}$, and Andrey Litvinov ${ }^{1}$ \\ ${ }^{1}$ Peter The Great St. Petersburg Polytechnic University, Department of Quantum electronics, \\ 29 Polytechnicheskaia str., Saint-Petersburg, 195251 Russia \\ ${ }^{2}$ Peter The Great St. Petersburg Polytechnic University, High school of software engineering, \\ 29 Polytechnicheskaia str., Saint-Petersburg, 195251 Russia
}

\begin{abstract}
Possibility of preparing of an alkali-atom's state with non-zero magnetic moment and zero spin-polarization was demonstrated in this work. There are zero average values of Pauli spin operators for all coordinates in the case. We suggest using a biharmonic laser field for preparing such states. Moreover both components of the laser field should be set to resonance with two sublines in D1 line of the alkali and have circular polarization. The amplitudes of the components needed to prepare the observed states was found. It is found interesting that polarization of the components of the laser may be orthogonal or parallel for preparing the states.
\end{abstract}

Generally, the spin polarization of alkali atoms is prepared with a laser field which has a circular polarization. In the case when a dark-state appears in the ground level the interaction between an atom in the dark-state and the laser field stops. Physically the darkstate is the Zeeman sublevel with the maximum value of the projection component of the atom's full angular momentum to the quantization axis. During the decay progress the atoms accumulate in this Zeeman sublevel. This leads to appearance of the non-zero expectation value for the atom spin operator or non-zero value of the spin-polarization. The atoms in this state have a magnetic moment which is determined by the gyromagnetic ratio of an electron and Lande factor of the energy level.

Preparing of the alkali atoms in the state with spin polarization is widely used in magnetometers, inertial navigation, experimental researches of spin-exchange interaction between atoms and in other $[1,2]$.

The goal of the work was observation of spin polarization pumping with two laser fields, which are set to resonance with two transitions in D1 line of the alkali atom. Scheme of pumping is demonstrated in Fig. 1. Notice, we made transition from the hyperfine-levels basis to the basis of electron and nuclear states because the relaxation of an electron-state and a nuclear-state passes with different velocities under collisions with the buffer. This detail led to essential amplification of the mathematical model in comparison with the usual semi-classical model. An assembly of an alkali was described with density matrix $\hat{\sigma}$, which involved all sublevels in the D1 line of the alkali and all transitions between this

Corresponding author: enp-tion@yandex.ru 
sublevels. Dynamics of atoms in semi-classical model was determined by the Liouville equation [3]:

$$
\frac{d \hat{\sigma}}{d t}=\frac{i}{\hbar}[\hat{\sigma}, \hat{H}]+\hat{\Gamma}_{r e l}\{\hat{\sigma}\}
$$

where $\hat{H}$ is Hamiltonian, $\hat{\Gamma}_{r e l}$ is relaxation superoperator which describes all processes of decay. We worked in dipole approximation:

$$
\hat{H}=\hat{H}_{0}-(\hat{\mathbf{d}} \cdot \mathbf{E}),
$$

where $\hat{H}_{0}$ is Hamiltonian without laser field, $\hat{\mathbf{d}}$ is an operator of atomic dipole, $\mathbf{E}$ is a vector of the electric field which contains both components of the laser pumping:

$$
\mathbf{E}=E_{1} \mathbf{l}_{1} e^{-i \omega_{1} t}+E_{2} \mathbf{l}_{2} e^{-i \omega_{2} t}+\text { c.c. } .
$$

In the equation (3) $E_{n}, \mathbf{l}_{\mathbf{n}}$ and $\omega_{n}$ are an amplitude, a polarization vector and a frequency of the $\mathrm{n}$-th component of the laser pumping.

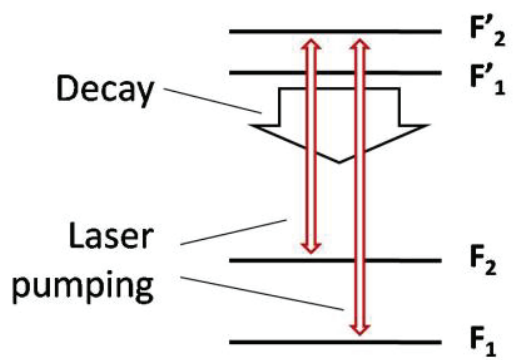

Fig. 1. Scheme of laser pumping of alkali's D1 line with biharmonic laser field

Further two regimes of laser pumping were observed: laser fields had the same polarizations $\left(\mathbf{l}_{\mathbf{1}} \mathbf{l}^{*}\right)=1$ and it had the orthogonal polarizations $\left(\mathbf{l}_{\mathbf{1}} \mathbf{l}_{\mathbf{2}}{ }^{*}\right)=0$. Variating amplitudes of the laser fields we found that spin polarization of the alkali became zero for certain values of the amplitudes. The most interesting fact is the effect was found for both regimes of pumping. We expected this effect for the second regime of the pumping, but reduction of spin polarization to zero for the same polarizations of laser fields surprised us.

In addition, we demonstrated that prepared to the state with zero spin polarization assembly of the alkali had non-zero expected value of the magnetic moment. In the case we couldn't use the Bloch's vectors for describing spin polarization of the alkali because a link between magnetization and spin polarization was not determined with an effective gyromagnetic ratio. We supposed that the considered states would have unusual magnetic properties and may be used in magnetometry, inertial navigation, medical physics.

The work supported by Russian Foundation for Basic Research (project No.16-32-00587)

\section{References}

[1] A.K. Vershovsky, A.S. Pazgalev, J. Tech. Phys. 76, 108 (2006)

[2] J.C. Fang, J. Qin, Sensors 12, 6331 (2012)

[3] W. Happer, Review of Modern Physics. 44, 170 (1972) 